\title{
First principles study of normal and fast diffusing metallic impurities in hcp titanium
}

\author{
N. Bernstein ${ }^{\mathrm{a}}$, A. Shabaev ${ }^{\mathrm{b}}$, S. G. Lambrakos ${ }^{\mathrm{a}, *}$ \\ ${ }^{a}$ Naval Research Laboratory, Washington, District of Columbia 20375, USA \\ ${ }^{b}$ George Mason University, Fairfax, Virginia 22030, USA
}

\begin{abstract}
The diffusivities of metallic impurities in hexagonal close packed (hcp) Ti vary widely, with some species showing normal diffusion and others diffusing anomalously fast. Based on a transition-state theory model for impurity diffusion mediated by vacancies, self, and impurity interstitials, we find that the diffusion rate will be limited primarily by the formation energies of the various defects. We use density functional theory to calculate these defect energies, including the formation energies of vacancies, self interstitials, and metallic impurities in substitutional and interstitial positions in hcp Ti. We find that for normal diffusers the vacancy mediated diffusion mechanism dominates, while for fast diffusers the dominant mechanism is hopping directly between interstitial sites. The activation energy for the latter mechanism is significantly lower than that for vacancy mediated diffusion, explaining the large diffusivities and low activation energies observed experimentally for these fast diffusing impurities.
\end{abstract}

\section{Introduction}

Titanium is an important material for structural applications because of its high strength, low weight, and corrosion resistance. For such applications Ti is alloyed with various other elements in order to improve its mechanical properties and achieve specific performance criteria. These alloying elements can remain in the bulk Ti lattice, which has a hexagonal close packed (hcp) structure at low temperatures, segregate at defects such as dislocations or grain boundaries, or affect its transformation to other crystal structures. Diffusion of the alloying elements, which can affect their interaction with the Ti lattice and its defects, shows a striking variability: while some alloying elements diffuse within $\mathrm{Ti}$ at rates similar to that of Ti itself, i.e., self-diffusion, others diffuse anomalously fast $[1,2,3]$. In crystalline solids diffusion is typically mediated by point defects, usually either vacancies or interstitials, which provide atoms with freedom to

\footnotetext{
*Corresponding author

Email addresses: noam.bernstein@nrl.navy.mil (N. Bernstein), ashabaev@gmu.edu (A. Shabaev), samuel.lambrakos@nrl.navy.mil (S. G. Lambrakos)
} 
move between lattice sites without incurring too large of an energy penalty [4]. A fundamental understanding of the diffusion of impurities in Ti is important for many of its applications. One example is friction-stir welding, a relatively low temperature solid-state joining technique, where the addition of a nickel foil between the two plates to be joined greatly improves the resulting weld quality [5]. The ability of the Ni atoms to change the properties of the material in the weld region despite the $\mathrm{Ti}$ and $\mathrm{Ni}$ being mixed only on relatively large length scales depends on the anomalously fast diffusion of Ni into the Ti lattice.

Impurity metal atoms in $\mathrm{Ti}$ can be divided into two groups with respect to their diffusivity: normal, including e.g. $\mathrm{Al}[6], \mathrm{Zr}[7,8]$, and $\mathrm{Au}[9]$, and fast, including e.g. Fe [10], Co [11], Ni [1], and $\mathrm{Cu}$ [12]. Since normal impurities diffuse at rates comparable to Ti self diffusion (about $10^{-17} \mathrm{~m}^{2} / \mathrm{s}$ at $T=1100 \mathrm{~K}$ ), which is believed to be vacancy mediated, it has been proposed that they also move by similar mechanisms $[2,3]$. The high mobility of fast metallic diffusers (about $10^{-14}-10^{-11} \mathrm{~m}^{2} / \mathrm{s}$ at $T=1100 \mathrm{~K}$ ), which in some cases is even higher than that of small non-metal atoms such as $\mathrm{C}, \mathrm{O}$, and $\mathrm{N}[13]$, has been attributed to an interstitial mediated mechanism $[1,2,3]$. The qualitative difference in diffusivity is correlated with several other properties $[2,14]$. Fast diffusing impurities diffuse faster parallel to the $c$ axis than in the perpendicular directions, while this ordering is reversed for normal impurities. They have smaller metallic radii and lower solubilities than normal impurities, a trend first noted by Hood for hcp Zr [14], and they are more likely to stabilize the $\beta$-Ti (body-centered cubic) high temperature phase or lead to amorphization of the structure [15]. The disparity in diffusivities has been linked with the expected difference between vacancy- and interstitial mediated diffusion $[1,2,3]$, but neither the microscopic reason for the dominance of each mechanism for a different set of elements, nor the empirically observed correlation with atomic size, have been fully explained. Neumann and Tuijn attributed the differences in diffusivity to solubility variations, which lead to variations in the fraction of impurities that are present as interstitials [16]. There have been many experimental studies of self and impurity diffusion in Ti, as reviewed in Refs. 2 and 3, but only a few relevant atomistic simulations have been published. The desire for chemically specific predictive information about different impurity elements requires the use of first principles electronic structure methods, such as density functional theory (DFT), which, unlike interatomic potentials, do not have to be parameterized for each combination of interacting species, but the large unit cells required make such calculations challenging. Most published simulations on Ti have considered only intrinsic defects $[17,18,19,20,21,22,23]$, non-metallic impurities [21], or the interaction between substitutional impurities and vacancies [24]. There have also been some DFT simulations comparing the structure and migration of interstitial Fe and $\mathrm{C}$ in the related hcp material Zr $[25,26]$, but none comparing different metal impurities.

One important observation from experiments on high purity samples is that the presence of even small concentrations of certain fast diffusers, e.g. $50 \mathrm{ppm}$ $\mathrm{Fe}$, can greatly increase the diffusivity of normal diffusers, and reduce the apparent activation energy of diffusion $[3,27,28,29,30]$. When these fast diffusers 
are present, the distinction between normal and fast diffusers remains, but the values of the diffusivities and activation energies for normal diffusers are significantly different from those of more pure material. Because so few experiments have been carried out on sufficiently pure samples, most data in the literature for normal diffusers is affected, and is therefore not quantitatively representative of the interaction between pure $\mathrm{Ti}$ and the normal diffusers. As a result, such experimental data on normal diffusers is not directly comparable to most atomistic simulations, including this work, which consider only pure Ti with a single impurity species.

Many models have been developed to relate microscopic processes and parameters to macroscopic diffusion kinetics in metals. Some are based on equilibrium thermodynamics and transition state theory, leading to diffusivities proportional to a product of a defect concentration and a mobility, which are exponential (through the Arrhenius equation) in a formation and migration energy, respectively [3, 16, 21, 31, 32]. Others explicitly include non-equilibrium effects through the Fick equation with kinetic coefficients coupling the concentrations and fluxes of various defect and impurity species [33, 34, 35, 36, 37].

Here we use first principles DFT simulations to calculate formation energies that govern the exponential temperature dependence of defect concentrations, and relate our calculations to the observed variation in impurity diffusivities. We study the relative energies of two native point defects, vacancies and selfinterstitials, and a number of impurity atoms at different sites. These include the normal diffusers $\mathrm{Ga}$, In, $\mathrm{Hf}, \mathrm{Nb}, \mathrm{Pb}, \mathrm{Sn}, \mathrm{Al}, \mathrm{Zn}, \mathrm{Zr}$, and $\mathrm{Au}$, and the fast diffusers, $\mathrm{Fe}, \mathrm{Co}, \mathrm{Ni}, \mathrm{Cr}, \mathrm{Mn}$, and $\mathrm{Cu}$, in a substitutional geometry and in several possible interstitial geometries. We use these defect formation energies in the context of a model for diffusivity that includes three diffusion mechanisms, and show that different migration mechanisms will dominate for the two classes of impurities: fast diffusers diffuse by hopping between interstitial sites, while normal diffusers hop between substitutional sites, mediated by vacancies or $\mathrm{Ti}$ self-interstitials.

In Secs. 2 and 3 we present the model for diffusion and a brief descriptions of the computational methods and model systems, respectively. In Sec. 4 we present results of simulations for the minimum energy defect structures for native defects and for impurities. In Sec. 5 we discuss our results and their implications for impurity diffusion mechanisms, and summarize in the final Section.

\section{Model}

Atomic transport in crystalline materials has been studied for many years to develop descriptions of macroscopic properties from details of the atomic jumps in the lattice. $[3,16,20,21,31,32,33,34,35,36,37,38,39]$ There are three main mechanisms for impurity diffusion [39], which combine additively to give a total diffusivity. When the impurity is in a substitutional position, an adjacent defect is required to enable diffusion with a sufficiently low barrier for the motion to be observable in experimental time scales and temperatures. Two 
types of point defects can serve this function: vacancies can mediate impurity diffusion when they diffuse through the substitutional impurity site, and so can self interstitials through a kick-out or interstitialcy mechanism. In addition, if the impurity has a stable interstitial configuration, there is a direct mechanism where the impurity diffuses between interstitial sites [21, 32, 34, 40]. The total diffusivity of impurity $X$ can be written as the sum of contributions from these three mechanisms

$$
D^{X}=D_{v}^{X}+D_{i \mathrm{Ti}}^{X}+D_{i X}^{X},
$$

where the subscript $v$ indicates the diffusion of substitutional $X$ mediated by vacancies, $i \mathrm{Ti}$ indicates the diffusion of substitutional $X$ mediated by $\mathrm{Ti}$ self interstitials, and $i X$ indicates the diffusion of interstitial $X$. This basic idea has been widely used when considering multiple diffusion mechanisms, whether or not such an equation is explicitly written. For the case of fast diffusers in $\mathrm{Ti}$, all three of the mechanisms we consider here have been considered, but not necessarily simultaneously. Here we assume that the defects are in equilibrium $[40,41]$, so their concentrations are

$$
e^{-E^{f} / k T}
$$

where $E^{f}$ is the defect formation energy, $k$ is Boltzmann's constant, and $T$ is the temperature, and their hopping is thermally activated with Arrhenius form

$$
A e^{-E^{m} / k T},
$$

where $E^{m}$ is the migration energy, and $A$ is a prefactor representing the effects of hop attempt frequency, correlation factor, and distance. As we discuss in more detail in Sec. 5, here we make the approximation that $A$ is independent of temperature. Figure 1 illustrates three paths of diffusion.

Figure 1a shows the path for the vacancy mediated migration. The vacancy contribution to diffusivity can be written as

$$
D_{v}=\frac{c_{s X}}{c_{X}} A_{v} e^{-E_{v}^{f} / k T} e^{-E_{v}^{m *} / k T},
$$

where $c_{X}$ is the total concentration of impurities, $c_{s X}$ is the concentration of substitutional ones, and the dependence on their ratio reflects the fraction of impurity atoms that are substitutional and available to migrate via this mechanism [16]. The formation energy dependence simply reflects the equilibrium concentration of vacancies, and the migration energy dependence reflects the temperature dependence of the rate at which vacancies hop through the lattice including correlation effects [38]. According to transition state theory, the effective migration energy is the highest barrier (relative to the energy of the vacancy in the bulk) along the path that the vacancy travels as it interacts with the impurity

$$
E_{v}^{m *}=\max \left(E_{v}^{m}, E_{v-X}^{m}\right),
$$

where $E_{v}^{m}$ is the bulk vacancy migration energy, and $E_{v-X}^{m}$ is the highest barrier for vacancy migration near a substitutional impurity $X$. Since the interaction 


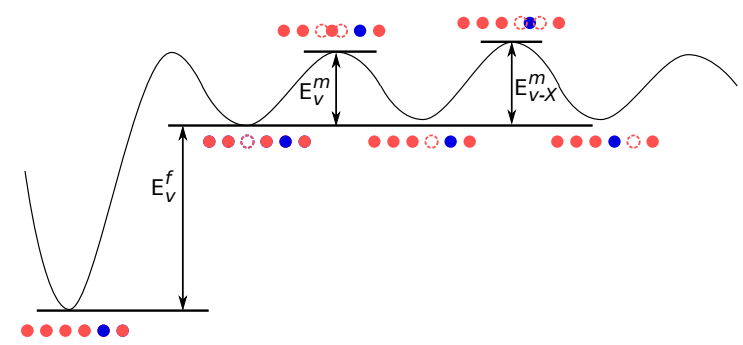

(a) Vacancy mediated migration

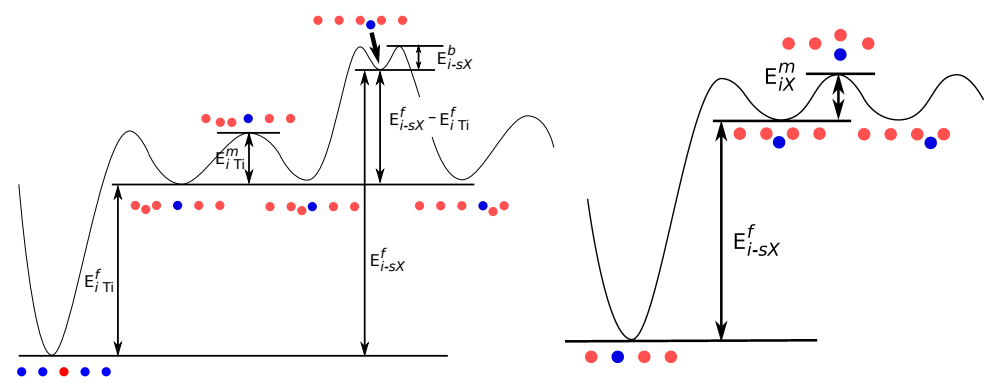

(b) Interstitial mediated kick-out mi- (c) Direct interstitial migragration tion

Figure 1: (color online) Diagrams for three mechanisms of diffusion showing relevant configurations and their energy levels. Light filled circles (red online) indicate host atoms, dark filled circles (blue online) indicate impurity atoms, and dashed circles indicate empty host lattice sites. (a) Vacancy mediated diffusion, showing the energy cost to form the vacancy $E_{v}^{f}$, barrier for migration of an isolated vacancy $E_{v}^{m}$, and barrier for exchange of a vacancy and adjacent impurity $E_{v-X}^{m}$. (b) Interstitial mediated kick-out migration, showing the energy cost to form a self-interstitial $E_{i \mathrm{Ti}}^{f}$, barrier for migration of an isolated self-interstitial $E_{i \mathrm{Ti}}^{m}$, energy cost for the self interstitial to kick out a substitutional impurity $X$ to an interstitial position $E_{i-s X}^{f}-E_{i \mathrm{Ti}}^{f}$, and barrier for this step $E_{i-s X}^{b}$. (c) Direct interstitial migration of an impurity $X$ showing the energy cost for transforming an impurity from a substitutional to an interstitial position, assuming that the vacancies or interstitials that mediate the reaction are in equilibrium, $E_{i-s X}^{f}$, and the barrier to the migration of an interstitial impurity $E_{i X}^{m}$. 
of the vacancy and the impurity is typically short ranged [33, 41, 42], $E_{v-X}^{m}$ is likely to differ from $E_{v}^{m}$ only when the vacancy is adjacent to the impurity, but since both are associated with the exchange of the vacancy with a neighboring atom, both are expected to be of the same order. It is also possible that this interaction energy can change the concentration of vacancies (or intrinsic interstitials dicussed below) near the impurity, an effect that we do not consider here.

The interstitial-mediated kick-out migration process is shown in Fig. 1b. The expression for the self interstitial contribution to diffusion $D^{i \mathrm{Ti}}$ is equivalent to Eq. 4 with the replacement of the vacancy-related quantities with corresponding ones for self interstitials. The only substantive difference is the nature of the transition state that controls the activation energy. For a self interstitial to facilitate the motion of a substitutional impurity, it must kick out the impurity to an intermediate (unstable or metastable) interstitial configuration, and itself move to a substitutional position. The interstitial impurity rapidly relaxes to a nearby substitutional position (different from the initial one), kicking out that host atom to form a new self interstitial. As for the vacancy, the effective migration energy is the maximum

$$
E_{i \mathrm{Ti}-X}^{m *}=\max \left(E_{i \mathrm{Ti}}^{m}, E_{i \mathrm{Ti}-X}^{m}\right),
$$

of the self interstitial migration energy, $E_{i \mathrm{Ti}}^{m}$, and the activation energy for the process where the self interstitial kicks out the substitutional impurity, $E_{i \mathrm{Ti}-X}^{m}$. Unlike in the case of the vacancy these are qualitatively different pathways, and we can estimate the activation barrier for the kick-out mechanism as

$$
\begin{aligned}
E_{i \mathrm{Ti}-X}^{m} & =E_{i X}^{f}-\left(E_{s X}^{f}+E_{i \mathrm{Ti}}^{f}\right)+E_{i-s X}^{b} \\
& =-E_{i \mathrm{Ti}}^{f}+E_{i-s X}^{f}+E_{i-s X}^{b},
\end{aligned}
$$

where $E_{i X}^{f}$ is the formation energy of the interstitial impurity, $E_{s X}^{f}$ is the formation energy of the substitutional impurity, $E_{i \mathrm{Ti}}^{f}$ is the formation energy of the self interstitial, $E_{i-s X}^{f}=E_{i X}^{f}-E_{s X}^{f}$, and $E_{i-s X}^{b}$ is a non-negative additional barrier energy. If the interstitial impurity site is metastable the additional barrier will be positive. Our calculations give us the three formation energies that contribute to $E_{i \mathrm{Ti}-X}^{m}$, but because we do not calculate the additional barrier, we have only a lower bound on the overall migration energy. If we substitute this migration energy expression into the expression for the self-interstitial-mediated diffusivity, we get

$$
\begin{aligned}
D_{i \mathrm{Ti}} & =\frac{c_{s X}}{c_{X}} A_{i \mathrm{Ti}} e^{-E_{i \mathrm{Ti}}^{f} / k T} e^{-E_{i \mathrm{Ti}-X}^{m} / k T} \\
& =A_{i \mathrm{Ti}} e^{-\left(E_{i-s X}^{f}+E_{i-s X}^{b}\right) / k T},
\end{aligned}
$$

where the dependence on $E_{i \mathrm{Ti}}^{f}$ is cancelled out. Since the migration energy of the self interstitial is expected to be small, it is unlikely to be the maximum in Eq. 6. 
Finally, the direct hopping of impurities between interstitial sites in Fig. 1c leads to a diffusivity

$$
D_{i X}=A_{i X} \frac{c_{i X}}{c_{X}} e^{-E_{i X}^{m} / k T}
$$

which is proportional to the fraction of impurities that are interstitial $c_{i X} / c_{X}$, and therefore available to move via this mechanism. If we assume that the substitutional and interstitial impurity concentrations are in equilibrium with each other and with either vacancies or self interstitials (which are themselves in equilbrium with some defect sources and sinks, e.g. grain boundaries or dislocation cores) through either of the reactions

$$
\begin{gathered}
X_{s}+\mathrm{Ti}_{i} \leftrightarrow X_{i} \\
X_{i}+V \leftrightarrow X_{s},
\end{gathered}
$$

we find that the resulting interstitial-substitutional impurity concentration ratio is [31]

$$
\frac{c_{i X}}{c_{s}}=e^{-E_{X_{i}}^{f}-E_{X_{s}}^{f} / k T}=e^{-E_{X_{i-s}}^{f} / k T} .
$$

If the fraction of impurities that are interstitial is small (as the calculated formation energies we show below confirm), $c_{S X} \approx c_{X}$, and we can write the diffusivity as

$$
D_{i X}=A_{i X} e^{-E_{i-s X}^{f} / k T} e^{-E_{i X}^{m} / k T},
$$

where $E_{i X}^{m}$ is the migration energy of the interstitial impurity $X$, which is expected t o be small.

We can now combine the three contributions from Eqs. 4, 8, and 13 (again assuming that $c_{s X} \approx c_{X}$ ) to get

$$
\begin{aligned}
D^{X}= & A_{v} e^{-\left(E_{v}^{f}+E_{v *}^{m}\right) / k T}+ \\
& A_{i \mathrm{Ti}} e^{-\left(E_{i \mathrm{Ti}}^{f}+E_{i \mathrm{Ti}}^{m}\right) / k T}+ \\
& A_{i X} e^{-\left(E_{i-s X}^{f}+E_{i X}^{m}\right) / k T},
\end{aligned}
$$

if the self-interstitial-mediated mechanism is rate limited by the hopping of the self interstitial, or

$$
\begin{aligned}
D^{X}= & A_{v} e^{-\left(E_{v}^{f}+E_{v *}^{m}\right) / k T}+ \\
& A_{i \mathrm{Ti}} e^{-\left(E_{i-s X}^{f}+E_{i-s X}^{b}\right) / k T} \\
& A_{i X} e^{-\left(E_{i-s X}^{f}+E_{i X}^{m}\right) / k T}
\end{aligned}
$$

if it is limited by the exchange of the self and impurity interstitials. Because computing the prefactor is beyond the scope of this work, we assume here that the prefactors of all three mechanisms are approximately equal. Since the physics of bonding for all of our impurities is similar, and the lattice in which the atoms diffuse is also similar (the hcp lattice itself for the vacancy, and the lattice of low 
energy interstitial sites for the self and impurity interstitials), this is a reasonable approximation. We also ignore differences in the migration energies of the vacancy, self interstitial, and impurity interstitials, as well as the $X_{i-s}$ exchange barrier $E_{i-s X}^{b}$. This is probably a more severe approximation, since unlike the prefactors the migration energies enter the rate expressions exponentially, and since interstitial migration is believed to have a lower energy than vacancy migration $[22,23]$. However, as we show below the scale of all of the migration energies, $\leq 0.5 \mathrm{eV}$, is significantly less than the variation among the relevant formation energies, and we therefore expect the neglected variation in migration energies to have no qualitative effect on our results.

\section{Methods}

The calculations were performed using DFT with the Perdew-Burke-Enzerhoff generalized-gradient approximation [43] and the projector-augmented wave (PAW) $[44,45]$ method as implemented in the Vienna Ab-Initio Simulation Package (VASP) code $[46,47,48,49]$. The following configurations were treated as valence electrons: Ti $3 \mathrm{p}^{6} 3 \mathrm{~d}^{2} 4 \mathrm{~s}^{2}$, Cr $3 \mathrm{p}^{6} 3 \mathrm{~d}^{5} 4 \mathrm{~s}^{1}$, Mn $3 \mathrm{p}^{6} 3 \mathrm{~d}^{5} 4 \mathrm{~s}^{2}$, Fe $3 \mathrm{p}^{6} 3 \mathrm{~d}^{6} 4 \mathrm{~s}^{2}$, Co $3 \mathrm{p}^{6} 3 \mathrm{~d}^{7} 4 \mathrm{~s}^{2}$, Ni $3 \mathrm{p}^{6} 3 \mathrm{~d}^{8} 4 \mathrm{~s}^{2}$, Cu $3 \mathrm{p}^{6} 3 \mathrm{~d}^{10} 4 \mathrm{~s}^{1}$, Ga $3 \mathrm{~d}^{10} 4 \mathrm{~s}^{2} 4 \mathrm{p}^{1}$, In $4 \mathrm{~d}^{10} 5 \mathrm{~s}^{2} 5 \mathrm{p}^{1}$, Hf $5 \mathrm{p}^{6} 5 \mathrm{p}^{6} 5 \mathrm{~d}^{2} 6 \mathrm{~s}^{2}, \mathrm{Nb} 4 \mathrm{p}^{6} 4 \mathrm{~d}^{4} 5 \mathrm{~s}^{1}$, Pb $5 \mathrm{~d}^{10} 6 \mathrm{~s}^{2} 6 \mathrm{p}^{2}$, Sn $4 \mathrm{~d}^{10} 5 \mathrm{~s}^{2} 5 \mathrm{p}^{2}$, Al $3 \mathrm{~s}^{2} 3 \mathrm{p}^{1}$, Zn $3 \mathrm{~d}^{10} 3 \mathrm{~s}^{2}$, Zr $4 \mathrm{~s}^{2} 4 \mathrm{p}^{6} 4 \mathrm{~d}^{2} 5 \mathrm{~s}^{2}$, Au $5 \mathrm{~d}^{10} 6 \mathrm{~s}^{1}$. Note that despite the computational cost PAWs with extra valence electrons were chosen where available because their results differed significantly from the default PAWs, which are expected to be less accurate for the short bond lengths in the interstitial geometries. The plane-wave basis-set cutoff was set to $400 \mathrm{eV}$. For each element $X=\mathrm{Ti}$, Cr, $\mathrm{Mn}, \mathrm{Fe}, \mathrm{Co}, \mathrm{Ni}, \mathrm{Cu}, \mathrm{In}, \mathrm{Ga}, \mathrm{Nb}, \mathrm{Hf}, \mathrm{Sn}, \mathrm{Al}, \mathrm{Zn}, \mathrm{Zr}$, and $\mathrm{Au}$, bulk calculations were performed to determine the chemical potential $\mu_{X}$ defined as the total energy per atom. In these calculations the experimental lattice structures and parameters that were used are as follows: hcp Ti $a=2.95 \AA, c=4.68 \AA$, bcc Fe $a=2.87 \AA$, hcp Co $a=2.51 \AA, c=4.07 \AA$, fcc Ni $a=3.52 \AA$, fcc Cu $a=3.61 \AA$, fcc Al $a=4.05 \AA$, hcp $\operatorname{Zr} a=3.23 \AA, c=5.15 \AA$, and fcc Au $a=4.08 \AA$. For all materials except $\mathrm{Ti}$, the bulk calculations were performed with primitive cells for the corresponding lattice structures and $7 \times 7 \times 5$ (for hcp) or $7 \times 7 \times 7$ (for fcc and bcc) Monkhorst-Pack [50] $k$-point meshes.

All Ti calculations used an orthorhombic supercell consisting of $5 \times 3 \times 3$ duplicates of a unit cell with lattice vectors ${ }^{1}$ 
five slightly offset positions. In terms of the labels defined in Fig. 2, these high symmetry positions are the center of the tetrahedron 1-2-3-5, the center of the octahedron 2-5-6-3-4-7, the center of the basal plane triangle 1-2-5 separating two tetrahedra ( $\mathrm{tt}$ face), the center of the basal plane triangle 2-5-6 separating two octahedra (oo face), and center of the triangle 2-3-5 separating an octahedron and a tetrahedron (ot face). Since high symmetry positions will be either local minima or local maxima, starting near but not at such positions will allow the relaxation algorithm to find relevant local minima. Convergence with respect to self-consistency was iterated until the total energy difference between iterations was less than $10^{-6} \mathrm{eV}$ and relaxation continued until all atomic forces were less than $0.01 \mathrm{eV} / \AA$.

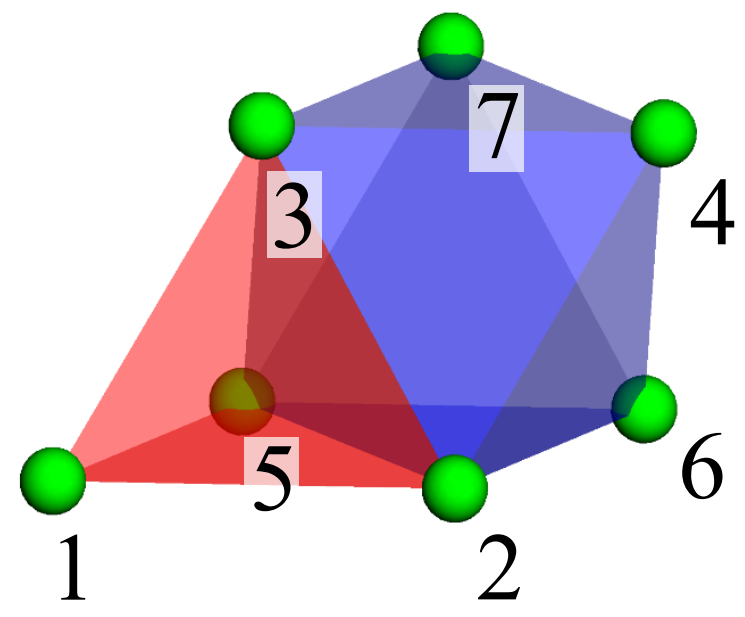

Figure 2: (color online) Visualization of the hep structure decomposed into tetrahedral (red, 1-2-3-5) and octahedral (blue, 2-5-6-3-4-7) volumes. Two adjacent basal planes contain 1-2-5-6 and 3-4-7. All basal plane triangles are shared by either two tetrahedra (tt face) (e.g. 1-2-5) or two octahedra (oo face) (e.g. 2-5-6). All triangles oblique to basal plane (e.g. 2-3-5) are shared by a tetrahedron and an octahedron (ot face).

\section{Results}

In Table 1 we list formation energies for various defects in the hcp-Ti lattice. For vacancies, the formation energy is defined as $E_{v}^{f}=E_{(N-1) \mathrm{Ti}}-(N-1) \mu_{\mathrm{Ti}}$, where $\mu_{\mathrm{Ti}}$ is the chemical potential $\mu_{X}(X=\mathrm{Ti})$ discussed in the previous section and $E_{(N-1) \mathrm{Ti}}$ is the relaxed energy of a cell with $N-1$ hcp-lattice Ti atoms and one vacancy. Our vacancy formation energy results are in agreement with other theoretical predictions [17, 20, 22], which are higher than the vacancy formation energy measured in experiment $[51,52,53]$. Similarly, the formation 
energy of an interstitial defect of element $X$ is $E_{i X}^{f}=E_{N T i+X}-N \mu_{\mathrm{Ti}}-\mu_{\mathrm{X}}$, where $E_{N \mathrm{Ti}+X}$ is the relaxed energy of a cell with $N$ Ti atoms in the hcp lattice and an interstitial $X$ atom, and the formation energy of a substitutional defect of element $X$ is $E_{\mathrm{s} X}^{f}=E_{(N-1) \mathrm{Ti}+X}-(N-1) \mu_{\mathrm{Ti}}-\mu_{\mathrm{X}}$, where $E_{(N-1) \mathrm{Ti}+X}$ is the relaxed energy of a cell with $N-1$ Ti atoms in the hcp lattice and a substitutional $X$ atom on the remaining hcp site. Negative substitutional formation energies correspond to exothermic dissolution. While the structures of vacancy and substitutional defects maintain the symmetry of the hcp lattice, each relaxed interstitial structure is different. For each element, the lowest relaxed energy of all the initial positions tested is listed, and the relaxed structure is described.

Table 1: Calculated properties of native and impurity defects in hcp Ti: defect formation energies in $\mathrm{eV}$ ( $E_{v}^{f}$ for vacancies, $E_{s X}^{f}$ for substitutionals, $E_{i X}^{f}$ for interstitials), metallic radius at coordination 12 in $\AA\left(r_{\text {met }}\right)$, whether the element is a fast diffuser in experiment $[2,3,7,8,9,12,28,54,55,56]$, and brief description of lowest energy geometry (details in Sec. 4). Except for Ti which is listed first, elements are in order of increasing metallic radius, which is nearly perfectly correlated with interstitial formation energy.

\begin{tabular}{lcccccc} 
& & \multicolumn{4}{c}{ fast } & geom. \\
\hline Elem. & $E_{v}^{f}$ & $E_{s X}^{f}$ & $E_{i X}^{f}$ & $r_{\text {met }}$ & diffuser? & oo face \\
$\mathrm{Ti}$ & 2.01 & & 2.36 & 1.47 & $\mathrm{n}[2,3,28]$ & $\sim(10 \overline{1} 0)$ \\
$\mathrm{Ni}$ & & -0.21 & 1.04 & 1.24 & $\mathrm{y}[2,3,8,28]$ & $\sim(\overline{1} 010)$ \\
$\mathrm{Co}$ & & 0.10 & 0.89 & 1.25 & $\mathrm{y}[2,3,8,28]$ & $\sim$ oo face \\
$\mathrm{Fe}$ & & 0.37 & 0.95 & 1.26 & $\mathrm{y}[2,3,28]$ & $\sim$ oo face \\
$\mathrm{Mn}$ & & 0.59 & 1.18 & 1.27 & $\mathrm{y}[2,8,28]$ & $(10 \overline{1} 0)$ \\
$\mathrm{Cu}$ & & 0.01 & 1.81 & 1.28 & $\mathrm{y}[12]$ & $\sim$ octa. ctr. \\
$\mathrm{Cr}$ & & 0.94 & 1.85 & 1.29 & $\mathrm{y}[2,8,28]$ & $(10 \overline{1} 0)$ \\
$\mathrm{Zn}$ & & -0.38 & 1.84 & 1.36 & & $(10 \overline{1} 0)$ \\
$\mathrm{Ga}$ & & -1.11 & 1.32 & 1.40 & $\mathrm{n}[3,28]$ & $(10 \overline{1} 0)$ \\
$\mathrm{Al}$ & & -0.98 & 1.66 & 1.43 & $\mathrm{n}[2,3,8,28]$ & $\sim(10 \overline{1} 0)$ \\
$\mathrm{Au}$ & & -0.92 & 1.52 & 1.44 & $\mathrm{n}[9,8,12]$ & $\sim(10 \overline{1} 0)$ \\
$\mathrm{Nb}$ & & 0.32 & 2.70 & 1.46 & & $(10 \overline{1} 0)$ \\
$\mathrm{In}$ & & -0.39 & 2.57 & 1.58 & $\mathrm{n}[3,28]$ & $(10 \overline{1} 0)$ \\
$\mathrm{Hf}$ & 0.14 & 3.05 & 1.59 & $\mathrm{n}[8,55,56]$ & $\sim(10 \overline{1} 0)$ \\
$\mathrm{Zr}$ & 0.21 & 3.01 & 1.60 & $\mathrm{n}[7,8]$ & $(10 \overline{1} 0)$ \\
$\mathrm{Sn}$ & -1.20 & 1.82 & 1.63 & $\mathrm{n}[54]$ & $(10 \overline{1} 0)$ \\
$\mathrm{Pb}$ & 0.04 & 3.07 & 1.70 & $\mathrm{n}[8]$ &
\end{tabular}

The formation energy of the self-interstitial is $E_{i \mathrm{Ti}}^{f}=2.36 \mathrm{eV}$, in good agreement with previous calculations [20,23], and higher than the vacancy formation energy $E_{v}^{f}$ by a relatively small difference of $0.35 \mathrm{eV}$. The lowest energy configurations were examined for $X=\mathrm{Fe}, \mathrm{Mn}, \mathrm{Co}, \mathrm{Cr}, \mathrm{Ni}, \mathrm{Cu}, \mathrm{Zn}, \mathrm{Nb}, \mathrm{Ga}, \mathrm{Au}, \mathrm{Al}$, $\mathrm{In}, \mathrm{Zr}, \mathrm{Hf}, \mathrm{Pb}$, and $\mathrm{Sn}$. The geometries of the lowest energy relaxed interstitial configuration for each elment are shown in Figs. 3 and 4. The relaxed interstitial positions can be roughly grouped into 5 configurations, as indicated in Table 1. 
The self interstitial, described as 'oo face,' is in the center of a basal plane oo triangle. Two other fast diffusers described as ' $\sim$ oo face' are near the oo face center, but slightly displaced from the basal plane, and the one described as ' $\sim$ octa. ctr.' is slightly displaced toward the basal plane from the octahdron center. Most other elements are described as ' $(10 \overline{1} 0)$ ' or ' $\sim(10 \overline{1} 0)$,' indicating that they are split along that crystallographic direction, with the impurity near a tt face and the Ti near an oo face, or tilted slightly away from that direction. One impurity, Co, described as '( 1010$)$ ' is also split along the same direction, but the impurity is near the oo face, and the Ti near the tt face. Because it is essential for determining the diffusivies due to the direct interstitial mechanism and to the interstitial-mediated kick-out mechanism, we calculate $E_{i-s X}^{f}=E_{i X}^{f}-E_{i, X}^{s}$, and plot the values in Fig. 5. We see that the fast diffusers tend to have a low value for this energy, while the normal diffusers have a high value, as compared with the vacancy formation energy.

Note that for all these elements, the reaction with a vacancy in Eq. 11 has the energy

$$
E_{s X}^{f}-\left(E_{v}^{f}+E_{i X}^{f}\right)<0,
$$

so it is energetically favorable for impurity interstitials to combine with vacancies and become substitutional. The reaction with a self-interstitial in Eq. 10 has the energy

$$
E_{s X}^{f}+E_{i}^{f}-E_{i X}^{f},
$$

which does not have the same sign for all impurities. For normal diffusers it is less than zero, indicating that it is energetically favorable for such interstitials to become substitutional by kicking out a Ti atom, making a self interstitial. Fast diffusers, on the other hand, have a positive energy difference for this reaction, indicating that they will be more stable in interstitial positions, in the absence of other defects such as vacancies.

\section{Discussion}

Our calculations indicate that the vacancy formation energy in $\mathrm{Ti}$ is about $2 \mathrm{eV}$, in good agreement with previous calculations [17, 20,22], but significantly higher than the experimental value of $1.2-1.55 \mathrm{eV}[51,52,53]$. The low experimental value is hard to reconcile with the measured diffusion activation energy in ultra pure samples of $3.14 \mathrm{eV}$ [3], which is expected to be the sum of the vacancy formation and vacancy migration energy. If both these experimental values are correct, the migration energy would have to be about $1.54-1.59 \mathrm{eV}$, a range that is not only much higher than calculations [22], but also unreasonably large compared to the formation energy itself. Possible explanations for this discrepancy are experimental error in the vacancy formation energy measurements, or a strong effect on the vacancy formation energy by impurities, similar to that seen in diffusivity activation energy measurements.

If, as is commonly assumed, Ti self diffusion is vacancy mediated, the activation energy of $2.0 \mathrm{eV}+0.5 \mathrm{eV}=2.5 \mathrm{eV}$ is somewhat lower than, but perhaps 
$\mathrm{Ni}$

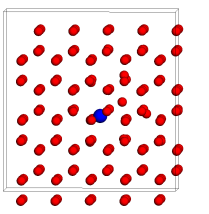

- $0 \circ \cdot 0$

- 0

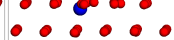

- 0.0

$\circ \bullet \circ .0$
Co

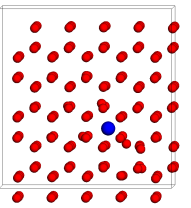

- 0 - 0

0.00

- 0.0

०. 0.0

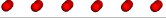

$\mathrm{Cu}$
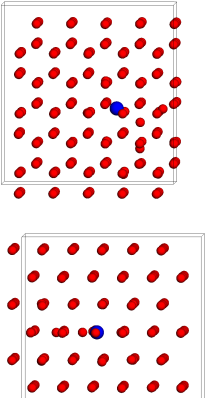

$\mathrm{Fe}$
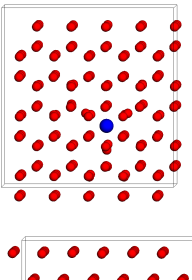

- 0 - 0

- $0 \circ$

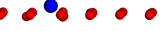

०. 0.0

Mn

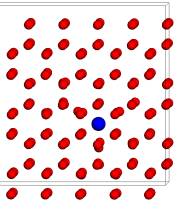

- 10 -

- $\bullet \bullet \bullet$

- 0.

- 0.0

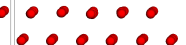

$\mathrm{Cr}$
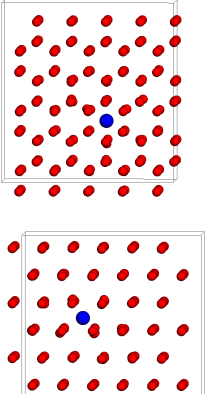

Figure 3: (color online) Visualization of the structure of the lowest energy relaxed interstitial configuration for each fast diffusing impurity element, viewed

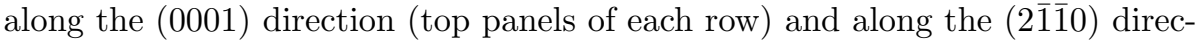
tion (bottom panels of each row). Small light (red online) spheres indicate Ti, and large dark (blue online) spheres indicate impurities. 
$\mathrm{Ti}$

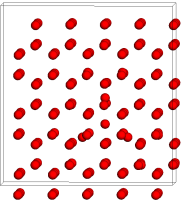

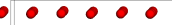

- ० ०००

- $\infty$ a .

- .0 .0

- 0.0

0.0 .0

$\mathrm{Au}$

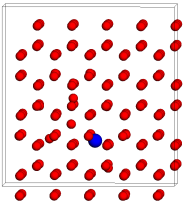

- ० ० ० ०

- ०. ०.

- $-\infty \cdot 0$

- ०. ०.

- $\bullet \bullet \bullet$

- .0 .0

$\mathrm{Zr}$

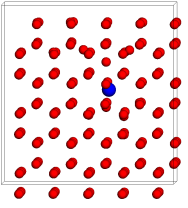

- $\bullet \bullet$

- ००००

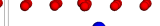

- $00 \infty$

- ००००

- ०. ०
$\mathrm{Zn}$

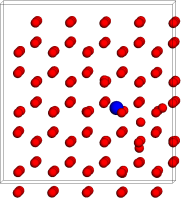

- ० • • ०

- $\bullet \circ \circ \circ$

- ० ०० ०

- $\infty \bullet \bullet \bullet$

- ००००

10.0 .0

$\mathrm{Nb}$

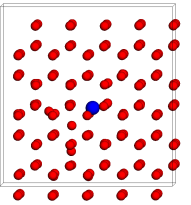

$\bullet \bullet \bullet \bullet \bullet$

- ०. ०.

- 0 - 0

- 0.0

- 0.0

Sn
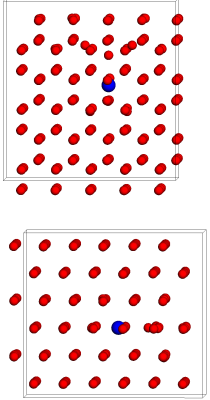

Ga

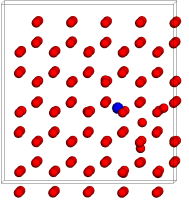

- $\bullet \bullet \bullet \bullet$

- ० ०००

- ००००

- $\bullet \bullet \bullet$

- $\bullet \bullet \bullet$

- .0.

In

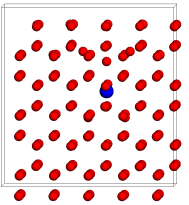

$\bullet \bullet \bullet \bullet \bullet$

००००.

- 0 - 0

0.000

Hf

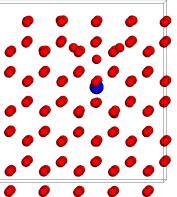

00

- 10.

- ००००

- 0 - 0

- ०. ०

- $\bullet \circ$.

Figure 4: (color online) Visualization of the structure of the lowest energy relaxed interstitial configuration for each normal diffusing impurity element. Orientation and notation same as Fig. 3. 
still in reasonable agreement with, experiment [17]. Interestingly, however, since the self interstitial formation energy is $2.4 \mathrm{eV}$, if it has a lower migration energy than the vacancy, as has been observed for other hcp metals [23], it could have a comparable activation energy and therefore make a significant contribution to self diffusion. Previous publications of calculated interstitial formation energies in $\mathrm{Ti}[23,20]$ do not seem to have considered this implication.

One of the simplifying assumptions we used in deriving our model is that the fraction of impurities that are interstitial is small. This fraction is simply

$$
\frac{c_{i X}}{c_{X}}=e^{\left(-E_{i-s X}^{f} / k T\right)},
$$

and we plot this effective formation energy in Fig. 5. For all elements we considered, this energy is significantly higher than $k T$, which is about $0.1 \mathrm{eV}$ for $T=1200 \mathrm{~K}$. Confirming our assumption, the largest interstitial fraction is therefore about $7 \%$, for $\mathrm{Fe}$ and $\mathrm{Mn}$ which have an interstitial-substitutional energy difference of about $0.5 \mathrm{eV}$. This result differs significantly from Neumann and Tuijn's model, which suggested that fast diffusers would have a much larger fraction of impurities in interstitial sites. However, unlike our work, they did not assume that the vacancies that facilitate the transition between interstitial and substitutional impurities are in thermodynamic equilibrium.

The diffusion of the impurities can proceed by three mechanisms: substitutional impurities can migrate aided by vacancies or self interstitials, or the impurities can migrate as interstitials. Within our model these three mechanisms add to give the total diffusivity, and the diffusion associated with each mechanism is controlled by an effective formation energy and a migration barrier, as in Eqs. 14 and 15. We assume here that the prefactors are comparable, and so the diffusivity variations will be dominated by the exponential dependence on the activation energies. Due to this exponential dependence, if one mechanism has a significantly lower energy (compared to the temperature) than the others, it is expected to dominate. For normal diffusers, we see in Fig. 5 that the vacancy formation energy is lower than $E_{i-s X}^{f}$ which controls direct interstitial diffusion, so the vacancy mediated mechanism will dominate. For fast diffusers we see the opposite relation, so direct migration as interstitials, with a correspondingly lower activation energy and higher diffusivity, will dominate. Interstitial-mediated motion through the kick-out mechanism will not change this trend. If the self interstitial migration barrier dominates, its formation energy will enter the rate expression (Eq. 14), and will be similar to vacancy mediated diffusion. If, on the other hand, the Ti-impurity interstitial exchange step is rate limiting, the rate will be controlled by the same energy (Eq. 15), $E_{i-s X}^{f}$, as for the direct interstitial migration mechanism.

Note that the preceding discussion ignored variation in the prefactor, as well as in the migration and barrier energies. We have not calculated these energies here, but previously published results range from about $0.2-0.3 \mathrm{eV}$ for self interstitial migration [23] to $0.53 \mathrm{eV}$ for vacancy migration [22]. The prefactors for self-interstitial- and vacancy-mediated diffusion, $A_{i \mathrm{Ti}}$ and $A_{v}$, can also vary due to a temperature dependence of the correlation factors, resulting 
in a change of the effective activation energy of the order of fractions of an $\mathrm{eV}[57,58]$. These are all considerably smaller than the variation in the difference between the energies for the two dominant mechanisms $\left(E_{i-s X}^{f}\right.$ and $\left.E_{v}^{f}\right)$ plotted in Fig. 5, so they are unlikely to change the trends, although they may change some details.

While our results reproduce the general trend of normal and fast diffusion, a more quantitative comparison with experimentally measured diffusivities or diffusion activation energies is difficult. Because of the known effect of even small amounts of certain impurities on measured diffusivities, in particular of normal diffusers [3, 28], little experimental data is quantitatively reliable enough to compare to these simulations, which are simplified in considering only Ti-single impurity interactions. While this is particularly true of the activation energies, as shown in Figs. 3 and 5 of Ref. 3, at high temperatures the differences between the lower and higher purity results are relatively small. We therefore plot the experimental diffusivity at $T=1100 \mathrm{~K}$ published in Refs. $2,7,8,56,3,54$ in Fig. 5. We see that the elements divide into two groups by diffusivity, with a substantial gap. Normal diffusers have diffusivities that are within two orders of magnitude of the Ti self-diffusivity, while fast diffusers have diffusivities that are at least 5 orders of magnitude higher. This trend in experimental diffusivities is well correlated with the formation energy difference between substitutional and interstitial positions for the impurity $E_{i-s X}^{f}$, which we have identified as controlling its diffusion according to our model. Fast diffusers have $E_{i-s X}^{f}$ that is lower than either the vacancy or self-interstitial formation energies, while normal diffusers have $E_{i-s X}^{f}$ that is higher. The lowest activation energy mechanism will dominate diffusion, and our model and calculations thus explain the variation in experimental diffusivities. The deviation from the overall trend, e.g. the very high diffusivities of $\mathrm{Co}$ and $\mathrm{Ni}$, is likely to be due to some of the factors we neglect as mentioned above, such as migration barriers, defect-defect interactions, and prefactor variations including both correlation and vibrational entropy effects.

While here we focused on the interplay between isolated substitutional and interstitial geometries for the impurities, defect-defect interactions can certainly modify these results. The effects include deviations from the dilute limit, as well as effective formation energies (modified by the binding energy) for intrinsic defects in the vicinity of impurities. An analysis of these more complicated configurations and mechanisms, in particular the possibility of mobile impuritydefect complexes, would be an interesting future extension of this work.

\section{Summary}

We have calculated the formation energies for vacancies, self interstitials, and substitutional and interstitial impurities in hcp Ti. Based on a model for diffusivity that includes three mechanisms, we find that the impurities can be divided into two groups. For elements that are normal diffusers experimentally our calculations show that the dominant mechanism with the lowest activation energy is that of vacancy mediated diffusion. For elements that are fast diffusers 
experimentally the lowest activation energy we find is that of direct migration as interstitials, which can be substantially lower than the vacancy formation energy that controls normal diffusion. Further work on the prefactors and migration barriers will be important to fully explain the interesting and technologically important diffusion processes in this material.

Acknowledgement. NB and SGL acknowledge funding for this work from the Office of Naval Research (ONR) through the Naval Research Laboratory's basic research program.

\section{References}

[1] G. M. Hood, R. J. Schultz, Phil. Mag. 26 (1972) 329-336.

[2] H. Nakajima, M. Koiwa, ISIJ International 31 (1991) 757-766.

[3] Y. Mishin, C. Herzig, Acta Mater. 48 (2000) 757-766.

[4] P. Haasen, Physical Metallurgy, 2nd Edition, Cambridge University Press, Cambridge, 1986.

[5] R. K. Everett, J. N. Wolk, A. Shabaev, S. G. Lambrakos, Issues concerning small additions of ni to ti friction stir welds, in: T. DeRoy, S. A. David, T. Koseki, H. Basdeshia (Eds.), Trends in Welding Research 2012: Proceedings of the 9th International Conference, June 48, 2012, Chicago, Illinois, USA, ASM International, 2013, pp. $842-847$.

[6] M. Köppers, C. Herzig, M. Friesel, Y. Mishin, Acta Mater. 45 (10) (1997) 4181-4191.

[7] R. A. Pérez, F. Dyment, H. Matzke, G. Linker, H. Dhers, J. Nucl. Mater. 217 (1994) 48-53.

[8] R. A. Pérez, M. L. Aguirre, F. Dyment, J. Nucl. Mater. 229 (1996) 15-23.

[9] J. H. R. dos Santos, P. F. P. Fichtner, M. Behar, R. Perez, F. Dyment, Appl. Phys. A 58 (1994) 453-457.

[10] H. Nakajima, M. Koiwa, S. Ono, Scr. Metall. 17 (1983) 1431-1434.

[11] E. Santos, F. Dyment, Phil. Mag. 31 (4) (1975) 809-827.

[12] O. Taguchi, Y. Iijima, Phil. Mag. A 72 (6) (1995) 1649-1655.

[13] C. Herzig, Y. Mishin, S. Divinski, Metall. Mater. Trans. A 33 (2002) 765775 .

[14] G. M. Hood, J. Phys. F: Metal Phys. 8 (6) (1978) 1677-1689.

[15] A. L. Greer, N. Karpe, J. Bøttiger, J. Alloy. Compd. 194 (1993) 199-211.

[16] G. Neumann, C. Tuijn, Physica B 315 (2002) 164-170. 
[17] G. Vérité, F. Willaime, C. C. Fu, Solid State Phenom. 129 (2007) 75.

[18] O. Le Bacq, F. Willaime, A. Pasturel, Phys. Rev. B 59 (1999) 8508.

[19] X. L. Han, Q. Wang, D. L. Sun, H. X. Zhang, Scr. Mater. 56 (2007) 77.

[20] A. T. Raji, S. Scandolo, R. Mazzarello, S. Nsengiyumva, M. Härting, D. T. Britton, Philos. Mag. 89 (2009) 1629.

[21] A. T. Raji, R. Mazzarello, S. Scandolo, S. Nsengiyumva, M. Härting, D. T. Britton, Phys. Rev. B 83 (2011) 054120.

[22] S. L. Shang, L. G. Hector Jr., Y. Wang, Z. K. Liu, Phys. Rev B 83 (2011) 224104 .

[23] G. Vérité, C. Domain, C. C. Fu, P. Gasca, A. Legris, F. Willaime, Phys. Rev. B 87 (2013) 134108.

[24] Q. M. Hu, D. S. Xu, D. Li, Phil. Mag. A 81 (2001) 2809-2821.

[25] R. A. Pérez, M. Weissmann, J. Nucl. Mater. 374 (2008) 95-100.

[26] R. C. Pasianot, R. A. Pérez, V. P. Ramunni, M. Weissmann, J. Nucl. Mater. 392 (2009) 100-104.

[27] C. Herzig, T. Wilger, T. Przeorski, F. Hisker, S. Divinski, Intermet. 9 (2001) 431-442.

[28] R. A. Pérez, H. Nakajima, F. Dyment, Mater. Trans. 44 (1) (2003) 2-13.

[29] G. M. Hood, J. Nucl. Matter. 135 (1985) 292-295.

[30] W. Frank, Phil. Mag. A 63 (1991) 897-913.

[31] F. C. Frank, D. Turnbull, Phys. Rev. 104 (1956) 617-618.

[32] C.-C. Fu, F. Willaime, Phys. Rev. B 72 (2005) 064117.

[33] A. R. Allnatt, A. B. Lidiard, Rep. Prog. Phys. 50 (1987) 373-472.

[34] J. L. Bocquet, Phil. Mag. A 63 (1991) 157-183.

[35] A. Barbu, A. B. Lidiard, Phil. Mag. A 74 (1996) 709.

[36] E. Meslin, C.-C. Fu, A. Barbu, F. Gao, F. Willaime, Phys. Rev. B 75 (2007) 094303.

[37] A. R. Allnatt, I. V. Belova, G. E. Murch, Phil. Mag. 94 (2014) 2487-2504.

[38] A. B. Lidiard, Phil. Mag. A 46 (1955) 1218-1235.

[39] P. M. Fahey, P. B. Griffin, J. D. Plummer, Rev. Mod. Phys. 61 (1989) 289-384. 
[40] R. E. Howard, A. B. Lidiard, Rep. Prog. Phys. 27 (1964) 161-240.

[41] S. M. Murphy, J. Nucl. Muter. 168 (1989) 31-42.

[42] A. Seeger, K. P. Chik, phys. stat. sol. 29 (1968) 455-542.

[43] J. P. Perdew, K. Burke, M. Ernzerhof, Phys. Rev. Lett. 77 (1996) 3865.

[44] P. E. Blöchl, Phys. Rev. B 50 (1994) 17953.

[45] G. Kresse, D. Joubert, Phys. Rev. B 59 (1999) 1758.

[46] G. Kresse, J. Hafner, Phys. Rev. B 47 (1993) 558.

[47] G. Kresse, J. Hafner, Phys. Rev. B 49 (1994) 14251.

[48] G. Kresse, J. Furthmüller, Comput. Mat. Sci. 6 (1996) 15.

[49] G. Kresse, J. Furthmüller, Phys. Rev. B 54 (1996) 11169.

[50] H. J. Monkhorst, J. D. Pack, Phys. Rev. B 13 (1976) 5188.

[51] V. O. Shestopal, Sov. Phys. Solid State 7 (1966) 2798.

[52] I. I. Novikov, V. V. Roshchupkin, N. A. Semashko, L. K. Fordeeva, J. Eng. Phys. Thermophys. 39 (1981) 1316.

[53] E. Hashimoto, E. A. Smirnov, T. Kino, J. Phys. F 14 (1984) L215.

[54] R. A. Pérez, F. Dyment, M. Behar, Mater. Lett. 57 (2003) 2670-2674.

[55] M. Behar, F. Dyment, R. A. Perez, J. H. R. Dos Santos, R. L. Maltez, E. J. Savino, Phil. Mag. A 63 (5) (1991) 967-972.

[56] R. A. Pérez, F. Dyment, G. Garćia Bermúdez, H. Somacal, D. Abriol, J. Nucl. Mater. 207 (1993) 221-227.

[57] S. Divinski, M. Salamon, H. Mehrer, Phil. Mag. 84 (8) (2004) 757-772.

[58] I. V. Belova, H. Mehrer, G. E. Murch, Phil. Mag. 91 (28) (2011) 3727-3743. 


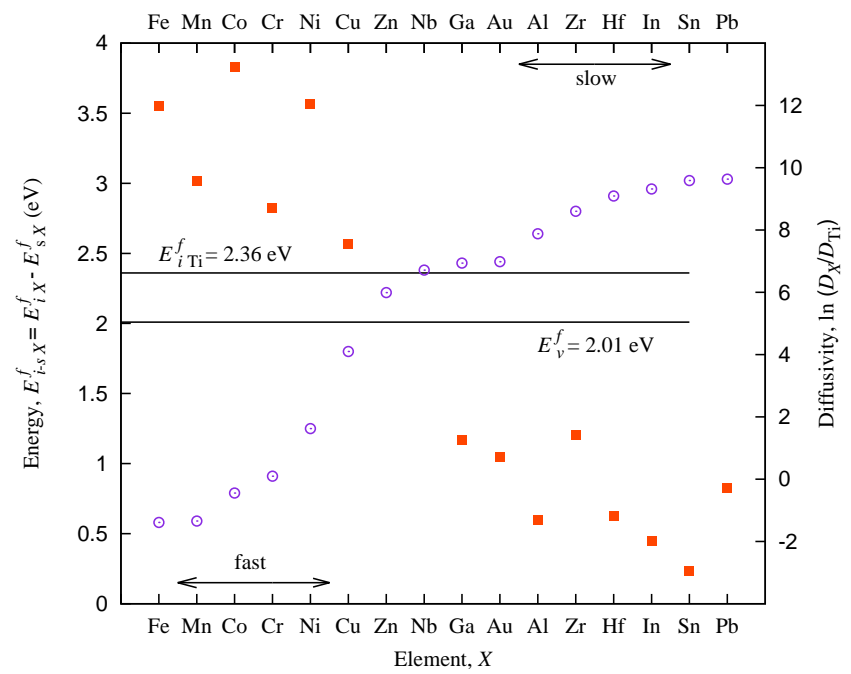

Figure 5: (color online) Impurity interstitial-substitutional formation energy difference (left axis, open symbols) and experimental diffusivity $[2,3,7,8,9$, $12,28,54,55,56]$ (scaled to the self-diffusivity) at $T=1100 \mathrm{~K}$ (right axis, filled symbols) for each element, in order of increasing formation energy difference. For comparison, vacancy and self interstitial formation energies, which account for most of the activation energies of vacancy and interstitial kick-out mediated diffusion, respectively, are indicated by solid lines. 
